\title{
Multidisciplinary Approach: An Essential Step in Diagnosing Granulomatous Disease
}

\author{
V. Sujanitha ${ }^{1}$, T. Kumanan ${ }^{1}$, G Selvaratnam ${ }^{1}$, S. Giridaran ${ }^{2}$ \\ Departments of ${ }^{1}$ Medicine, Teaching Hospital, Jaffna, Sri Lanka; ${ }^{2}$ Consultant Surgeon, Base Hospital, Jaffna, Sri Lanka.
}

\section{Corresponding Author:}

Dr. V. Sujanitha

Email: sujanitha@yahoo.com

This is an Open Access article distributed under the terms of the Creative Commons Attribution License (creativecommons.org/ licenses/by/3.0).

Received : August 24, 2017

Accepted : December 4, 2017

Published : December 5, 2017

\begin{abstract}
Background: Abdominal lymphadenopathy is a common presentation of many diseases and sarcoidosis should be considered as an important differential diagnosis. Prompt early diagnosis would help to minimize the disease progression and its complications. Case Report: A 51 year old female presented with appetite and weight loss of seven months duration and found to have an intra-abdominal lymphadenopathy. A celiac axis lymph node biopsy showed granulomatous lymphadenitis and the patient quickly progressed to develop hepato-splenomegaly with $\mathrm{CT}$ evidence of multiple attenuation areas in spleen, intra-pulmonary nodules with lymphadenopathy. An endo-bronchial biopsy also confirmed a non-necrotizing granulomatous inflammation. Patient had a remarkable improvement in symptoms with oral steroids. Conclusion: Intra-abdominal lymphadenopathy, an initial presentation of many common diseases has heterogeneous etiology and sarcoidosis is one important clinical diagnosis. Appropriate use of available investigations and careful regular clinical assessment with multi-disciplinary approach to identify new organ involvement will help to arrive at early diagnosis and treatment.
\end{abstract}

Keywords: Biopsy, Lymphadenopathy, Lymphadenitis, Sarcoidosis, Tuberculosis.

\section{Introduction}

Sarcoidosis, a multisystem disease affects all ethnic and racial group and all ages though the incidence is high among the age group of 20-39 years [1]. It is characterized by the presence of non-caseating granulomatous lesions. Lungs and lymphoid system are the most commonly involved organs. Intraabdominal sarcoidosis, especially intra-abdominal lymphadenopathy may mimic lymphoma, diffuse metastasis and granulomatous diseases [2]. Liver and spleen are the most common intra-abdominal sites of involvement of sarcoidosis. The etiology of sarcoidosis is unknown. Enlarged lymph nodes are detected in approximately in $30 \%$ of patients, particularly in porta-hepatis, para-aortic region and celiac axis [3].

\section{Case Report}

A 51 year old female presented to the surgical ward with the complaints of epigastric pain, appetite and weight loss of seven months duration. She had a past medical history of uncomplicated diabetes mellitus and was on oral hypoglycemic medications. Physical examination was unremarkable at presentation. Initial complete blood count was normal with a hemoglobin of $11.3 \mathrm{~g} / \mathrm{dL}$ (normal 12-15), total leukocyte count 8900 cells $/ \mathrm{mm}^{3}$ (normal value: $4000-11000$ ) with normal differential count and platelet count of 2.4 lakhs/ $\mu \mathrm{L}$ (normal: $1.5-4.5$ ). ESR was $10 \mathrm{~mm}$ in $1^{\text {st }}$ hour. Her liver biochemistry showed an isolated rise in alkaline phosphatase of 942 IU/L (normal: 44-150). Ultrasound imaging of the abdomen 
showed multiple para-aortic lymphadenopathy which was subsequently confirmed by contrast enhanced computed tomography with no evidence of other abdominal masses [Fig.1a]. Blood film was performed to see any features of primary hematological disorders however it showed marked rouleaux formation and mild eosinophilia. Her LDH and serum amylase levels were within normal limits.

In the context of marked constitutional symptoms such as significant loss of appetite and weight, gastro-duodenoscopy was performed and it revealed edematous mucosa in proximal stomach with the suspicion of an external compression and the histology showed mild chronic gastritis without activity and edema of lamina propria. Colonoscopy was normal. The treating surgical team opted for an exploratory laparotomy and found multiple enlarged lymph nodes without any other significant abnormality. Celiac axis lymph node was biopsied.

As the biopsy report of the celiac axis node was showing granulomatous lymphadenitis [Fig.2a], she was referred to the medical team for further evaluation. At this point, approximately a month has elapsed from the initial presentation; she had hepato-splenomegaly with persistent constitutional symptoms. Even though there were no respiratory signs a plain chest radiograph showed nodular opacities in the right base with a prominent right hilum. A repeat computed tomography of chest and abdomen showed multiple intra-pulmonary nodules involving inter-lobular septae and sub-pleural region bilaterally [Fig.1b]; enlarged bilateral para-tracheal, pre-tracheal, subcarinal, right-hilar and pre-aortic lymph nodes. Spleen was enlarged up to $13 \mathrm{~cm}$ with multiple attenuation areas; liver was enlarged with uniform enhancement without any focal lesion [Fig.1c].

In view of quickly progressing systemic involvement tuberculin skin test was done which was negative. A bronchoscopy showed nodular mucosa in right middle and lower lobe and endo-

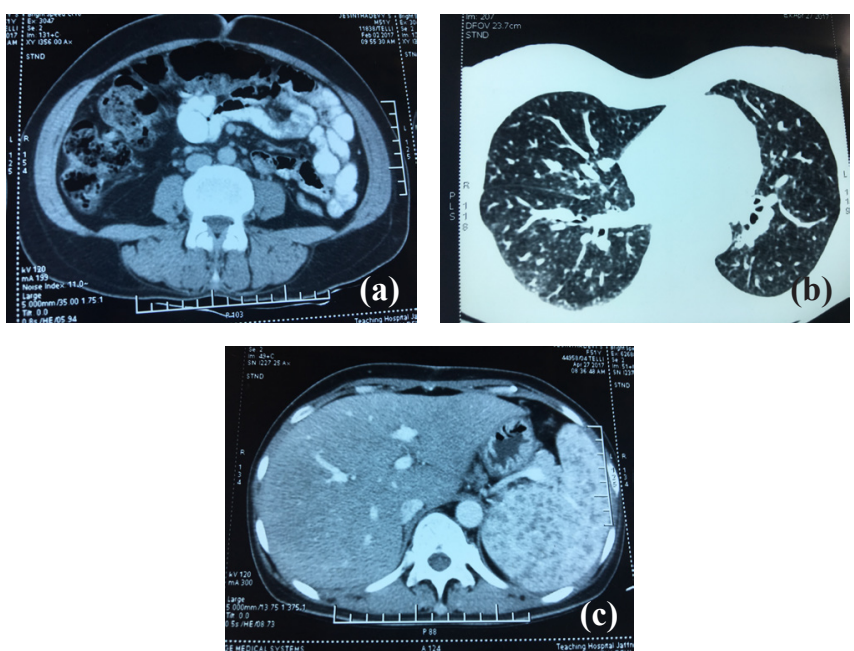

Fig.1(a): $C T$ abdomen showing multiple intra-abdominal lymphadenopathy. (b): HRCT of thorax showing bilateral nodular lesions mainly in the sub-pleural region. (c): CT abdomen showing multiple attenuated areas in spleen.

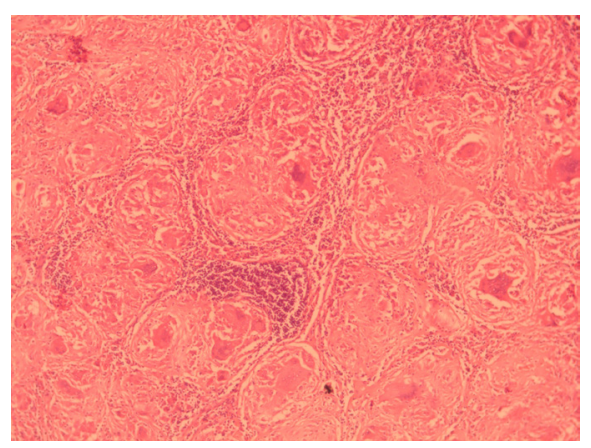

Fig.2(a): Histology of celiac lymph node showing nonnecrotizing granuloma.

bronchial biopsy was taken that revealed nonnecrotizing granulomatous inflammation. Fungal infection and tuberculosis were reasonably excluded from the extended culture. Serum calcium was $2.66 \mathrm{mmol} / \mathrm{L}$ (normal 2.2-2.7 $\mathrm{mmol} / \mathrm{L}$ ), and 24 hour urinary calcium level was $12.23 \mathrm{mmol} /$ day (normal: $<6.2 \mathrm{mmol} /$ day) which was well above the normal limits. Serum ACE level was $48.0 \mathrm{U} / \mathrm{L}$ (8-52) and 2D echocardiogram was normal.

Having excluded the most common infective causes of granulomata such as tuberculosis and possible neoplasm a likely diagnosis of sarcoidosis was made and treatment was initiated 
with oral prednisolone. With treatment patient has made a remarkable improvement in constitutional symptoms and repeat imaging is planned in next three months.

\section{Discussion}

Sarcoidosis is a chronic granulomatous condition of unknown origin. The diagnosis is based on histological findings of non-caseating granuloma in the presence of supportive radiological and clinical findings provided other common conditions are ruled out satisfactorily [4]. The pulmonary parenchyma and mediastinal and hilar nodal groups are the most commonly involved sites but any organ system can be involved. Hepatic involvement is seen in $75 \%$ of the cases in sarcoidosis, spleen being involved in $40-60 \%$ of cases mainly as splenomegaly or less commonly as multiple focal splenic lesions as in our case [5]. Most commonly intra-abdominal lymphadenopathy, splenic and hepatic sarcoidosis can be asymptomatic, but can present with marked constitutional symptoms as in our patient or sometimes pressure symptoms.

Intra-abdominal lymphadenopathy, being a common presentation of many diseases needs thorough evaluation. Malignancies in particular gastrointestinal tumors, hematological disorders and chronic infections need exclusion in the evaluation. SriLanka, being a tropical country, tuberculosis still remains the most common cause of intra-abdominal lymphadenopathy due to chronic infections. Other rare infections like histoplasmosis, coccidioidomycosis, and syphilis can also cause similar presentation. Intra-abdominal lymphadenopathy can be an initial presentation of sarcoidosis though it is rare.

Imaging plays a pivotal role in diagnosing sarcoidosis as in this patient. This patient had an isolated rise in alkaline phosphatase which is a common observation in sarcoidosis. An elevated 24 hour urinary calcium levels and a marginally high serum ACE levels further supported the diagnosis.
A negative tuberculin test and negative culture for tuberculosis and other chronic fungal infections excluded the common prevailing infections which mimic sarcoidosis. Typical radiological features and characteristic histological findings from two different sites further re-inforced the diagnosis of sarcoidosis.

Treatment of sarcoidosis is usually with corticosteroids with the other immunosuppressive therapy reserved for aggressive or recurrent diseases or steroid non responders [6]. American Thoracic Society and British Thoracic Society guidelines recommend corticosteroids for the initial progressive organ involvement in sarcoidosis [1]. Our patient was initiated on prednisolone $40 \mathrm{mg}$ daily and planned to continue for three months and to taper off with the assessment of clinical symptoms and planned for repeat imaging in three months.

\section{Conclusion}

Intra-abdominal lymphadenopathy, an initial presentation of many common diseases has heterogeneous etiology and sarcoidosis is one important clinical diagnosis. Appropriate use of available investigations and careful regular clinical assessment to identify new organ involvement will help to arrive a prompt early diagnosis and treatment. A multidisciplinary approach in complex clinical scenario like our patient will help to arrive at an early diagnosis and helps in patient management.

Acknowledgements: Authors acknowledge Dr. Anusha Ginige and Dr. N.D. Ranathunga, Department of Histopathology, Teaching Hospital Jaffna in providing histological slides and Dr. A. Murugananthan, Department of Parasitology Faculty of Medicine Jaffna in providing technical assistance in making images.

Contributors: VS and TK were involved in writing the manuscript; GS and SG helped in writing as well as edited the initial manuscript. VS will act as guarantor. All authors read and approved the final version of this manuscript.

Funding: None; Competing interests: None stated. 


\section{References}

1. Statement on sarcoidosis Joint Statement of the American Thoracic Society (ATS), the European Respiratory Society (ERS) and the World Association of Sarcoidosis and Other Granulomatous Disorders (WASOG) adopted by the ATS Board of Directors and by the ERS Executive Committee. Am J Respir Crit Care Med. 1999;160:736755.

2. Nunes H1, Bouvry D, Soler P, Valeyre D. Sarcoidosis. Orphanet J Rare Dis. 2007;19:46.
3. Warshauer DM, Molina PL, Hamman SM, Koehler RE, Paulson EK, Bechtold RE, et al. Nodular sarcoidosis of the liver and spleen: analysis of 32 cases. Radiology. 1995; 195:757-762.

4. Iannuzzi MC, Rybicki BA, Teirstein AS. Sarcoidosis. N Engl J Med. 2007;357:2153-2165.

5. Warshauer DM, Lee JK. Imaging manifestations of abdominal sarcoidosis. AJR Am J Roentgenol. 2004;182:15-28.

6. Gomez NS, Peters JI, Nambiar AM. Diagnosis and Management of sarcoidosis. Am Fam Physician. 2016;93:840-850. 